\title{
Colonic serrated pathway lesions: Molecular and histologic changes in serrated colonic proliferations (Review)
}

\author{
FRANZ FOGT $^{1}$, AMIR RAHEMTULLA ${ }^{2}$ and BO JIAN ${ }^{1}$ \\ ${ }^{1}$ Department of Pathology, University of Pennsylvania, Penn Presbyterian Medical Center, Philadelphia, PA; \\ ${ }^{2}$ Department of Pathology, Union Hospital, North Shore Medical Center, Lynn, MA, USA
}

Received October 29, 2007; Accepted December 3, 2007

\begin{abstract}
Serrated polyps of the intestinal tract, specifically those within the colon, represent a wide variety of benign and neoplastic changes. Diagnostic entities include benign hyperplastic changes in prolapse, hyperplastic polyps (HPs), serrated adenomas (SAs) and carcinomas with serrated morphology. Recent developments in molecular pathology have confirmed the existence of additional neoplastic growths, with potentially aggressive behavior, called sessile serrated adenomas (SSAs). It is important to be aware of the distinct aspects of clinical significance and the varying outcomes associated with different serrated changes. In this article, important histologic diagnoses with serrated histology are reviewed and juxtaposed, where applicable, with their respective molecular pathways.
\end{abstract}

\section{Contents}

1. Introduction

2. Serrated lesions, reactive

3. Hyperplastic polyps

4. Hyperplastic polyposis syndrome

5. Dysplastic serrated polyps

6. Sessile serrated adenoma

7. Serrated polyps in the vermiform appendix

8. Conclusion

\section{Introduction}

Serrated histology in biopsy specimens from colonic biopsies signifies the appearance of surface and crypt epithelium that

Correspondence to: Dr Franz Fogt, Department of Pathology, Penn Presbyterian Medical Center, University of Pennsylvania, 39th and Market Streets, Philadelphia, PA 19104, USA

E-mail: franz.fogt@uphs.upeun.edu

Key words: hyperplastic polyp, serrated adenoma, sessile serrated adenoma, serrated pathway protrude in a sawtooth pattern from the underlying stroma of the lamina propria, with or without histologically-verifiable nuclear dysplasia. The protruding epithelium demonstrates a significant proliferation of the epithelial aspect of the mucosa without adequate associated growth of the cell-bearing stroma. In such cases, the epithelium not only covers the lamina propria, but 'invades' the lumen to accommodate the cell mass. The diagnosis of serrated histology is, in most cases, straightforward and benign. It includes a wide range of benign and neoplastic diagnostic entities, including reactive changes in the prolapse, solitary rectal ulcer, inflammatory bowel disease and small hyperplastic polyps (HPs), which are not associated with dysplasia and neoplasia. On the other hand are the so-called serrated adenomas (SAs), which have serration and histologically-evident nuclear atypia of the epithelium, and the newly-described sessile serrated adenomas (SSAs), which have no histologic evidence of epithelial dysplasia. These latter lesions are neoplastic and can therefore develop a highgrade dysplastic and invasive phenotype. Of these lesions, the attention will be focussed on SSAs as they are difficult to consistently diagnose and recommendations for their followup need to be made.

\section{Serrated lesions, reactive}

It would appear that both the reactive processes and regenerative changes of colonic surface mucosa exhibit histological changes of hyperplasia (Fig. 1). In these cases, the glands are mildly dilated and have a mild-to-moderate sawtooth appearance. Usually, the typical slight thickening of the basement membrane seen in HPs is not apparent. In the case of prolapse, the presence of smooth muscle fibers within the lamina propria is also detectable, probably subsequent to the pulling of the folds through the peristalsis. A similar mecha-nism most likely causes the serrated appearance of small inflammatory polyps, such as those seen in the small poly-poid fragments from surveillance biopsies of patients with inflammatory bowel disease (Fig. 2). Larger HPs in patients with ulcerative colitis (UC) likely represent true HPs as 3p, p53, p16 and K-ras mutations have been shown to be similar in sporadic HPs and UC-associated polyps (1). In large inflammatory polyps, the surface mucosa is often absent, but the reactive and proliferative activity of crypt epithelium within the polyp has the morphology of hyperplastic glands. 


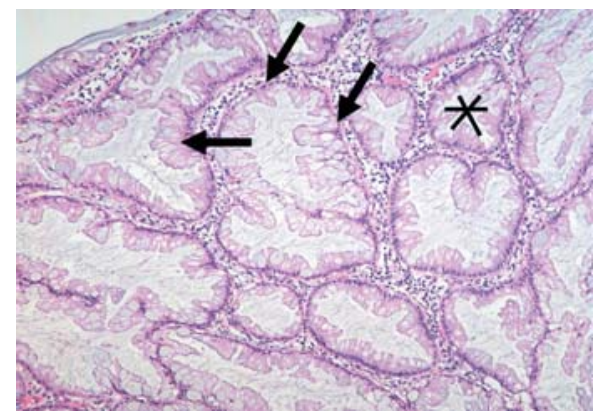

Figure 1. Serrated morphology and accumulation of histologically-benign epithelial cells starting to occupy the lumen of crypts, resulting in a sawtooth appearance of epithelial cells (arrows) and star-like cross sections of crypts (star)

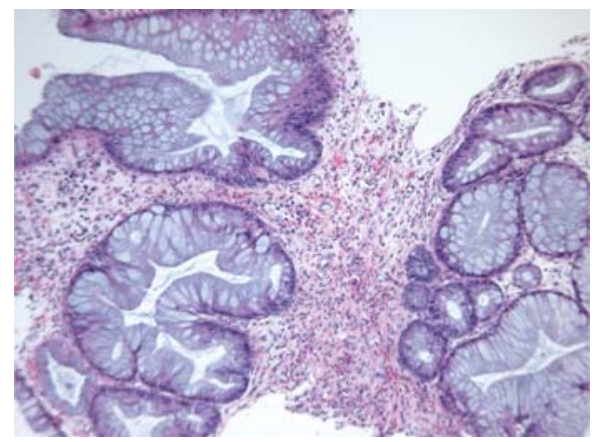

Figure 2. Serrated hyperplastic-type features in ulcerative colitis. The background of inflammatory cells suggests small inflammatory type polyp.

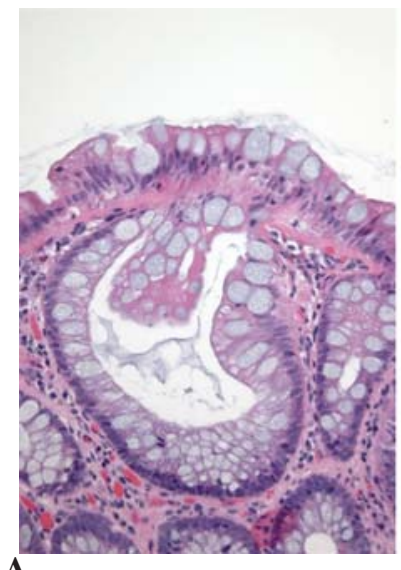

A

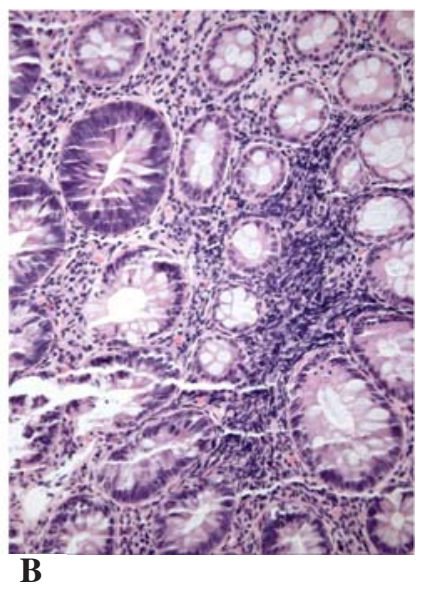

Figure 3. Aberrant crypt foci. (A) Serrated type ACF; (B) adenomatous ACF in patient with familial adenomatosis.

\section{Hyperplastic polyps}

The earliest histopathologically-recognizable elements of HPs represent aberrant crypt foci (ACF). HPs develop from non-dysplastic ACF (Fig. 3), as compared to dysplastic ACF in dysplastic polypoid lesions, such as adenomas (2). Traditionally regarded as non-neoplastic lesions, HPs are prevalent in $12-15 \%$ of colonoscopies and are found mostly in the left colon. They are usually quite small in size - typically $<5 \mathrm{~mm}$ - and represent $\sim 85 \%$ of all serrated polyps biopsied in routine endoscopies. Histologically, HPs are characterized

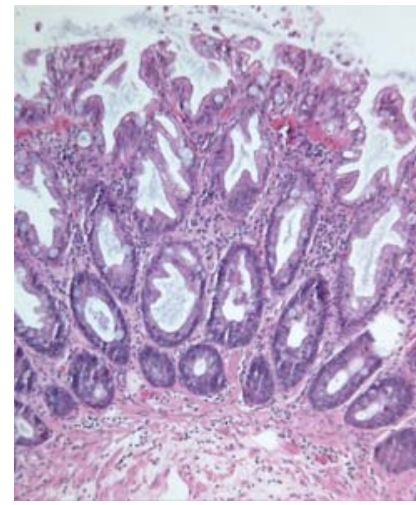

A

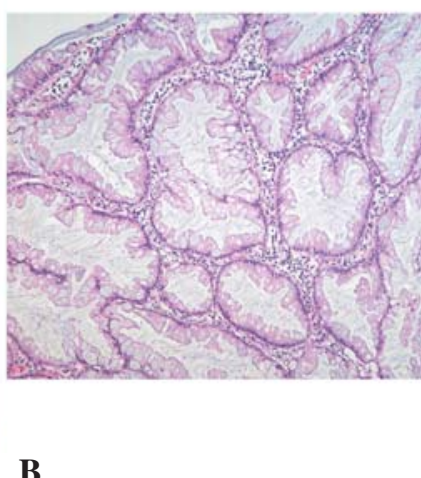

B
Figure 4. Hyperplastic polyp. (A) Serration limited to the upper 2/3 of the crypt. Straight basal aspects of crypts with deep proliferative cell pool (arrow). (B) Nuclei are basally located and bland.

by serration in the upper 1-2/3 of the crypt length and have a basally-located proliferative crypt pool with dense, nondysplastic nuclei (Fig. 4). Mutation studies have revealed K-ras and BRAF mutations in $29-75$ and $6 \%$ of cases, respectively. Mutational changes in p53 have not been observed in HPs. Methylation studies have been inconclusive with regards to changes in p16, MINT1 and HMLT1. However, SLC5A8 methylation has been observed in 22\% of HPs. SLC5A8 TSG, a member of the family of sodium solute transporter (3), appears to be associated with growth advantage in colon cancers as inactivation of SLC5A8 (usually via methylation) leads to growth advantage in the subset of colon cancers (4).

\section{Hyperplastic polyposis syndrome}

Hyperplastic polyposis has been described as a syndrome with familial clustering (5) associated with the development of colorectal carcinoma (CRC). The risk, for these patients, of developing CRC exceeds $50 \%$. The WHO definition of hyperplastic polyposis syndrome includes the presence of more than 30 HPs throughout the colon or more than 5 HPs proximal to the sigmoid, of which two are $>10 \mathrm{~mm}$ in size. The definition additionally includes patients with any number of HPs occurring proximal to the sigmoid colon who have a first-degree relative with hyperplastic polyposis syndrome. The development of CRC in patients with hyperplastic polyposis appears to involve four distinct pathways: the classical supressor gene pathway with APC involvement, the MSI high pathway with loss of hMLH1, the MSI low pathway with $\mathrm{O}^{6}$-methylguanine-DNA-methyltransferase (MGMT) methylation inactivation and, as $43 \%$ of cases present BRAF mutations, a BRAF-associated pathway (6).

\section{Dysplastic serrated polyps}

SAs were originally described by Longacre et al (7), replacing the term 'mixed hyperplastic adenomatous polyp' which was previously used (8). The initial description followed a retrospective study of some 18,000 polyps. The discovery of SAs was made in around $0.6 \%$ of those polyps. Two thirds of these had previously been categorized as HPs or adenomas. Of those lesions which fell into the SA category, $11 \%$ contained 


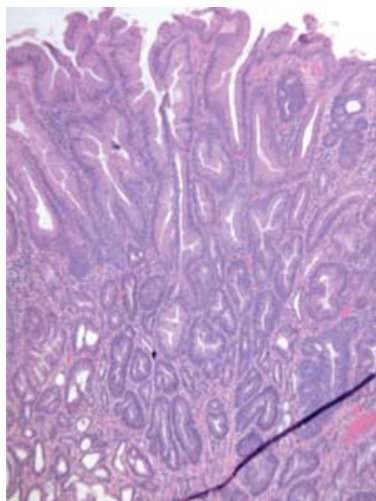

A

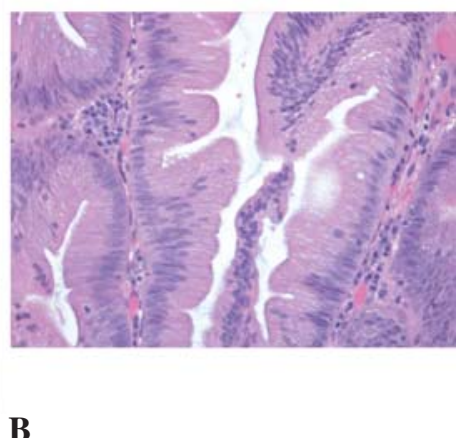

Figure 5. Serrated adenoma, (A) serration of polyp with prominent dysplastic cell lining. (B) Serrated cells show palisading, crowding and nuclear overlapping, histological correlate of dysplasia.
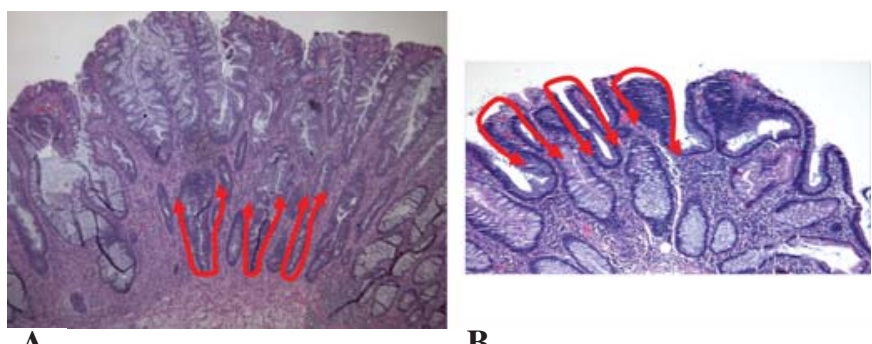

A

B

Figure 6. Proliferative zone (PZ). In serrated adenomas (A) the PZ is located basally, which represents a normal basal to luminal growth pattern. In tubular adenomas (B), the PZ is located at the top, which led to the conclusion that adenomas 'push' themselves outward into the lumen.

intramucosal carcinoma. Currently, it is assumed that SAs represent about $2-3.5 \%$ of polyps recovered during routine endoscopy sessions. The prevalent location is in the distal (left) colon and rectum. The gross appearance of SAs is that of pedunculated broad-based polyps. Histologically, there is serration of the epithelium with nuclei showing definitive changes of dysplasia, including nuclear overlapping, enlargement and irregularity, and eosinophilic cytoplasm (Fig. 5). Histologic evaluation of the proliferative cell pool in SA has shown that proliferation takes place within the basal aspect of the colonic crypts, similar to normal growth and the growth pattern of HPs, with an associated arrest in apoptosis along the crypts (9). This results in the overgrowth of epithelial cells which, in turn, results in a serrated histology pattern. This is in contrast to the superficial translocation of the proliferative pool in tubular adenomas (10), which leads to an inverted growth pattern whereby the proliferation of epithelial cells is directed towards the luminal side of the polyp with subsequent polypoid outgrowth of the proliferative cell pool into the lumen of the colon (Fig. 6) (11). SA have been observed in association with familial adenomatosis coli and in Cronkhite Canada syndrome, but are not usually associated with HNPCC carcinomas. Early investigations into the development of invasive carcinomas suggested that microsatellite instability drove HPs, via SA, to invasive tumors with a very high proliferative activity (12). These studies, however, may have used some polyps which belong to a recent newly-defined

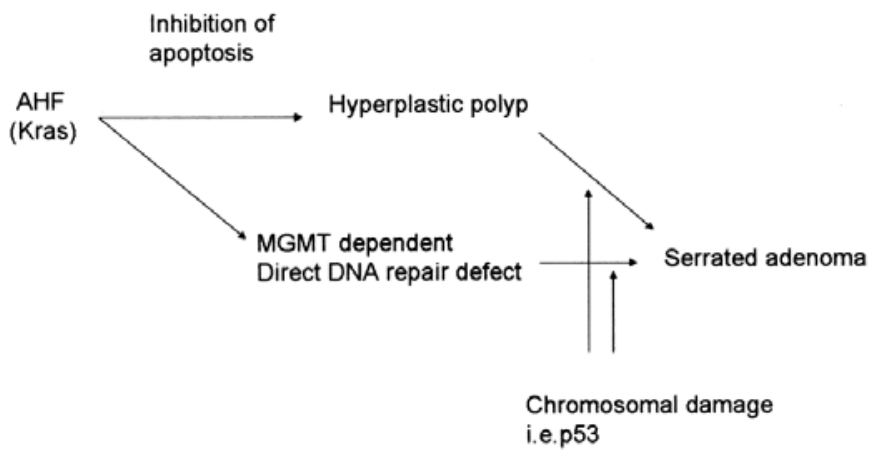

Figure 7. Serrated adenoma: distal colonic serrated pathway.

group, called SSAs (see below). The current assumption is that the average SA has a risk of malignant change similar to that of tubular adenomas.

Early mutation studies reported a low frequency of K-ras mutations in SA (13). However, once strict morphologic criteria were observed and SSAs were excluded, the majority of large rectal SAs harbored K-ras mutations $(14,15)$. Alterations in the p53 gene have also been reported, with controversial results. Hiyama et al reported p53 immunoreactivity in 100\% of their SAs, and confirmed mutations in $47 \%$ of cases (16). The same group later reported p53 alterations of only $29 \%$ in SA (17). Similarly, Rubio et al reported p53 alterations in a high percentage of SAs with high-grade dysplasia (18). Our own and other studies have also shown that p53 alterations are not significantly different from usual tubular adenomas $(6,10,12)$. It may be that there is, in fact, an increase of p53 alteration with increasing dysplasia in SAs. Telomerase activity, which is up-regulated in precancerous and invasive cell populations, is associated with the absence of wild-type p53 function: Oka et al showed that $56 \%$ of SAs, but only $4 \%$ of HPs, had overexpressed human telomerase reverse transcriptase (hTERT) which correlated closely to the degree of dysplasia and immunopositivity of p53 (17). Few methylation studies have been performed on SAs. It is apparent that there is infrequent methylation or loss (19) of hMLH1, RB1 or VHL, but a high frequency $(82 \%)$ of methylation in SLC5A8, similar to HPs (3). There is, however, an indication that the direct DNA repair mechanisms through the MGMT pathway are altered in SAs (20).

There appears to exist a distinct genetic pathway associated with left sided, distal colonic dysplastic serrated polyps, SAs, which starts the level of an atypical (hyperplastic) crypt focus with K-ras mutation. Inhibition of apoptosis may lead to the serrated aspect of the morphology. This ACF may develop into a hyperplastic type polyp, if no further aggregated damage occurs. If silencing of MGMT with loss of direct DNA repair mechanism accrues, a MSI-L serrated dysplastic polyp with potential for invasive phenotype may develop (Fig. 7).

\section{Sessile serrated adenoma}

The concept of SSA developed from the observation that patients developed carcinomas from HPs which were considered to represent entirely benign proliferations (21) and 


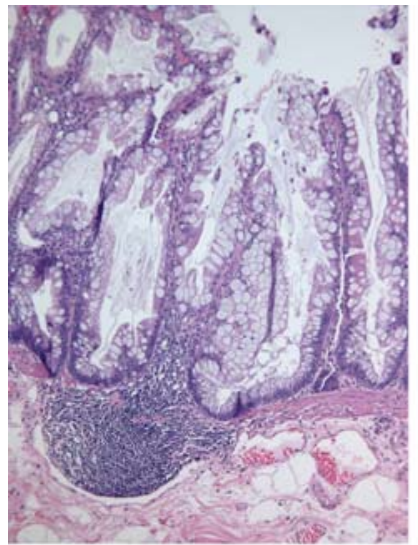

A

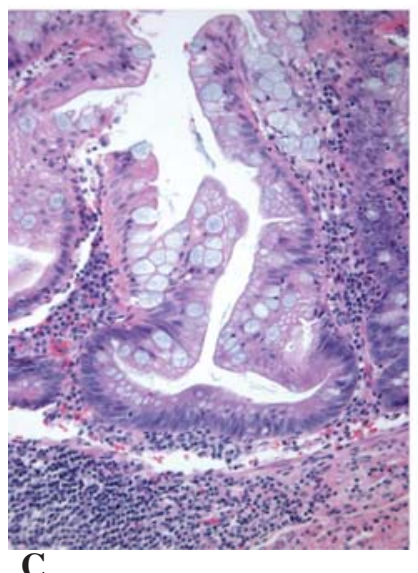

C

Figure 8. Sessile serrated ademoma: (A) hyperplastic type lining of crypts along the entire crypt length with dilatation of crypts at the basal aspect; (B) higher power of dilated crypt base. Sessile serrated ademoma: (C) higher power of dilated basal crypt; (D) hyperplastic type glands with almost entire absence of lamina propria between crypts.

from observations that, in some cases, hyperplastic morphology was observed to be closely associated with invasive CRCs (22). Due to the more proximal location of these lesions within the colon, the notion of a proximal serrated pathway was conceived. Further study of these polyps led to the ability to distinguish HPs with abnormal proliferation as distinct from usual HPs (23). These lesions are most frequently located in the proximal colon, are considered to represent a precursor lesion for invasive carcinoma and are designated as SSAs (24). It is assumed that these polyps show a stepwise progression from a purely serrated phenotype to a morphologically dysplastic phenotype, possibly in the form of mixed adenomatous-SSA polyps, towards an invasive phenotype (25). The semantic difficulties with this lesion rest in the notion of adenoma in a lesion that does not show the light microscopic features of dysplasia, as compared to typical colorectal adenomas and SAs. The use of the designation 'adenoma' as 'dysplasia' implies that it is dysplastic, i.e., genetically determined towards malignant transformation, a characteristic that most likely exists long before morphologic changes can be determined (26). The histologic criteria for the diagnosis of SSA have been previously published and appear to be easily applicable in daily practice $(25,27)$. In contrast to typical HPs, the serrated features of SSAs are not limited to their upper two thirds, but involve the entire length of the involved crypt. The base of the

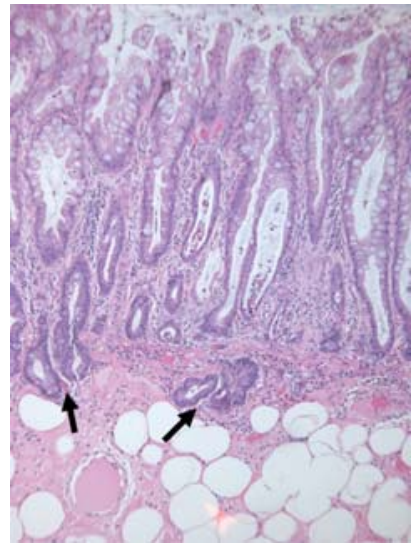

Figure 9. Sessile serrated adenoma with prolapse of glands into and through the muscularis mucoasae (arrow).

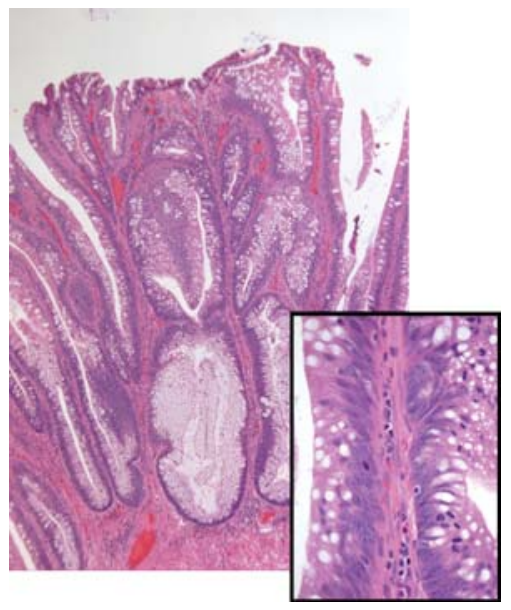

Figure 10. Sessile serrated adenoma with development of dysplasia. (A) Serrated architecture of polyp with characteristics of SSA and dysplastic nuclei (insert). (B) Staining for hMLH1. Loss of staining for MLH1 indication microsatellite instability.

crypt, cone shaped and proliferative in typical HPs, shows dilatation, bends, branching and horizontal features in SSAs lined by serrated epithelium (Fig. 8). In many cases, the lamina propria between serrated crypts is extremely narrow or virtually absent (Fig. 8D). The thickened basement membrane often seen in typical HPs is usually not noted. Some crypts are present within and slightly below the muscularis mucosae (Fig. 9). Molecular studies have shown that, like the other lesions discussed, SSAs develop from ACF precursor lesions (2). Genetic analysis has shown that, in contrast to the ACF of SAs and HPs, the ACF associated with SSAs develop with BRAF mutations. BRAF mutations are present in $\sim 75 \%$ of SSAs, but in only $20 \%$ of both HPs and SAs $(25,28,29)$. Immunohistochemical studies of SSA for microsatellite instability demonstrate frequent loss of 1hMLH1 (Fig. 10). Methylation studies confirmed methylation of hMLH1 in MSI-H cases in $~ 69 \%$ of cases, and methylation of MGMT in $20 \%$ of MSI-L cases. In addition to the molecular pathologic differences between SAs and SSAs, clinical and outcome data demonstrate differences between invasive CRCs ex SA vs. ex SSA. Serrated carcinomas of the SSA type, i.e., proximal serrated pathway, are associated with a 5-year survival of 


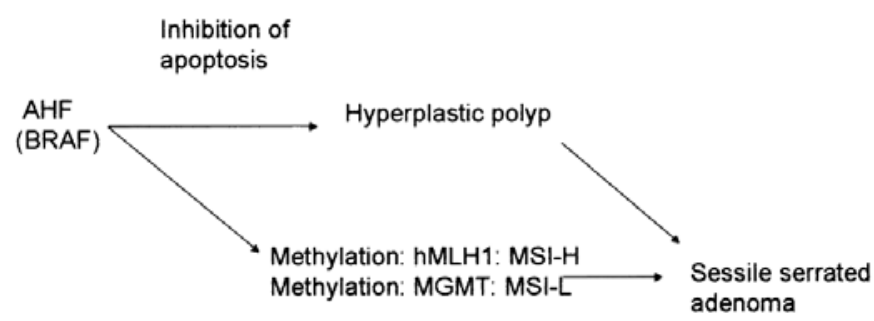

Figure 11. Sessile serrated adenoma: proximal colonic serrated pathway.

$\sim 70 \%$ vs. a $<30 \%$ survival in distal, SA-associated carcinomas. Similarly, proximal MSI-H tumors have a better prognosis compared to MSI-L distal cases. Proximal MSI-H cancers appear to not show additional treatment benefit from chemotherapy.

As with typical SAs, a specific proximal serrated pathway is responsible for the development of CRCs from SSA, which starts from the level of an atypical (hyperplastic) crypt focus with BRAF mutation. Again, the inhibition of apoptosis may lead to a serrated aspect of morphology. The ACF may develop into SSA either directly or through a stage of HP. Methylation of hMLH1 leads to an MSI-H invasive phenotype, whereas methylation of MGMT leads to an MSI-L phenotype (Fig. 11).

\section{Serrated polyps of the vermiform appendix}

A recent study of appendiceal serrated polyps demonstrated that they may show BRAF or KRAS mutations even if morphologically overt dysplasia is not seen. The molecular features of these polyps do not reliably identify morphologic subgroups in this location. However, as some polyps with overt dysplastic features may be associated with the clonal expansions of KRAS-mutated populations, the authors propose that serrated epithelial proliferations of the appendix be tentatively classified as nondysplastic or dysplastic serrated polyps until more information becomes available on their molecular features and biological significance (30).

\section{Conclusion}

Serrated lesions have previously been divided into benign hyperplastic type lesions without malignant potential and SAs with malignant potential. Clinical and histopathological observations have, however, shown that some lesions which were viewed as HPs may in fact be differentiated into lesions with varying tendencies to develop an invasive phenotype. The discovery of genetic pathways of proximal hyperplastic type lesions similar to potentially malignant SSAs has brought to light the fact that traditional ways of diagnosing disease may have to be reviewed when clinical evidence indicates doubts about specific premises. An important aspect of the diagnosis of SSAs is the fact that dysplasia is diagnosed, as implicated in the designation 'adenoma', without the histologic nuclear features typically associated with dysplasia. Through the ongoing genetic analysis of tissues from malignant lesions, precursor lesions of malignant disease and other changes - even those remote from malignant lesions - genetic aberrations have been observed which are associated with the development of tumors in histologically normal cells $(26,31,32)$ These findings indicate that the notion of dysplasia may have to be expanded, not only to morphologically evident nuclear changes, but also to genetic changes which are indicative of a definite change towards neoplastic phenotype in tissue cell populations.

\section{References}

1. Odze RD, Brien T, Brown CA, Hartman CJ, Wellman A and Fogt F: Molecular alterations in chronic ulcerative colitisassociated and sporadic hyperplastic polyps: a comparative analysis. Am J Gastroenterol 97: 1235-1242, 2002.

2. Makinen MJ: Colorectal serrated adenocarcinoma. Histopathology 50: 131-150, 2007.

3. Dong SM, Lee EJ, Jeon ES, Park CK and Kim KM: Progressive methylation during the serrated neoplasia pathway of the colorectum. Mod Pathol 18: 170-178, 2005.

4. Li H, Myeroff L, Smiraglia D, et al: SLC5A8, a sodium transporter, is a tumor suppressor gene silenced by methylation in human colon aberrant crypt foci and cancers. Proc Natl Acad Sci USA 100: 8412-8417, 2003.

5. Burt R and Jass JR: Hyperplastic polyposis. In: World Health Organization Classification of Tumours. Pathology and Genetics of Tumours of the Digestive System. Hamilton SR and Aaltonen LA (eds). IARC Press, Lyon, pp135-136, 2000.

6. Huang CS, O'Brien MJ, Yang S and Farraye FA: Hyperplastic polyps, serrated adenomas, and the serrated polyp neoplasia pathway. Am J Gastroenterol 99: 2242-255, 2004.

7. Longacre TA and Fenoglio-Preiser CM: Mixed hyperplastic adenomatous polyps/serrated adenomas. A distinct form of colorectal neoplasia. Am J Surg Pathol 14: 524-5237, 1990.

8. Urbanski SJ, Kossakowska AE, Marcon N and Bruce WR: Mixed hyperplastic adenomatous polyps - an underdiagnosed entity. Report of a case of adenocarcinoma arising within a mixed hyper-plastic adenomatous polyp. Am J Surg Pathol 8: 551-556, 1984.

9. Komori K, Ajioka Y, Watanabe H, Oda and Nimura Y: Proliferation kinetics and apoptosis of serrated adenoma of the colorectum. Pathol Int 53: 277-283, 2003.

10. Fogt F, Poremba C, Shibao K, Itoh H, Kohno K, Zimmerman RL, Gortz HG, Dockhorn-Dworniczak B, Urbanski SJ, Alsaigh N, Heinz D, Noffsinger AE and Shroyer KR: Expression of survivin, YB-1, and KI-67 in sporadic adenomas and dysplasia-associated lesions or masses in ulcerative colitis. Appl Immunohistochem Mol Morphol 9: 143-149, 2001.

11. Shiff SJ and Rigas B: Colon adenomatous polyps - do they grow inward? Lancet 349: 1853-1854, 1997.

12. Konishi K, Yamochi T, Makino R, Kaneko K, Yamamoto T, Nozawa H, Katagiri A, Ito H, Nakayama K, Ota H, Mitamura K and Imawari M: Molecular differences between sporadic serrated and conventional colorectal adenomas. Clin Cancer Res 10: 3082-3090, 2004.

13. Ajioka Y, Watanabe H, Jass JR, Yokota Y, Kobayashi M and Nishikura K: Infrequent K-ras codon 12 mutation in serrated adenomas of human colorectum. Gut 42: 680-684, 1998.

14. Nucci MR, Robinson CR, Longo P, Campbell P and Hamilton SR: Phenotypic and genotypic characteristics of aberrant crypt foci in human colorectal mucosa. Hum Pathol 28: 1396-13407, 1997.

15. Higashidani Y, Tamura S, Morita T, Tadokoro T, Yokoyama Y, Miyazaki J, Yang Y, Takeuchi S, Taguchi H and Onishi S: Analysis of K-ras codon 12 mutation in flat and nodular variants of serrated adenoma in the colon. Dis Colon Rectum 46: 327-332, 2003.

16. Hiyama T, Yokozaki H, Shimamoto F, Haruma K, Yasui W, Kajiyama $G$ and Tahara E: Frequent p53 gene mutations in serrated adenomas of the colorectum. J Pathol 186: 131-139, 1998.

17. Oka S, Tanaka S, Hiyama T, Kitadai Y, Yoshihara M, Shimamoto F, Haruma K and Chayama K: Human telomerase reverse transcriptase, $\mathrm{p} 53$ and $\mathrm{Ki}-67$ expression and apoptosis in colorectal serrated adenoma. Scand J Gastroenterol 37: 1194-1200, 2002.

18. Rubio CA and Rodensjo M: p53 overexpression in flat serrated adenomas and flat tubular adenomas of the colorectal mucosa. J Cancer Res Clin Oncol 121: 571-576, 1995.

19. Higuchi T, Sugihara K and Jass JR: Demographic and pathological characteristics of serrated polyps of colorectum. Histopathology 47: 32-40, 2005. 
20. Oh K, Redston M and Odze RD: Support for hMLH1 and MGMT silencing as a mechanism of tumorigenesis in the hyperplastic-adenoma-carcinoma (serrated) carcinogenic pathway in the colon. Hum Pathol 36: 101-111, 2005.

21. Torlakovic E and Snover DC: Serrated adenomatous polyposis in humans. Gastroenterology 110: 748-755, 1996.

22. Goldman H, Ming S and Hickock DF: Nature and significance of hyperplastic polyps of the human colon. Arch Pathol Lab Med 89: 349-354, 1970.

23. Torlakovic E, Skovlund E, Snover DC, Torlakovic G and Nesland JM: Morphologic reappraisal of serrated colorectal polyps. Am J Surg Pathol 27: 65-81, 2003.

24. Goldstein NS: Clinical significance of (sessile) serrated adenomas: another piece of the puzzle. Am J Clin Pathol 123: 329-330, 2005.

25. Snover DC, Jass JR, Fenoglio-Preiser C and Batts KP: Serrated polyps of the large intestine: a morphologic and molecular review of an evolving concept. Am J Clin Pathol 124: 380-391, 2005.

26. Zhuang Z, Vortmeyer AO, Mark EJ, Odze R, Emmert-Buck MR, Merino MJ, Moon H, Liotta LA and Duray PH: Barrett's esophagus: metaplastic cells with loss of heterozygosity at the APC gene locus are clonal precursors to invasive adenocarcinoma. Cancer Res 56: 1961-1964, 1996.
27. Sandmeier D, Seelentag W and Bouzourene H: Serrated polyps of the colorectum: is sessile serrated adenoma distinguishable from hyperplastic polyp in a daily practice? Virchows Arch 450: 613-618, 2007.

28. Kambara T, Simms LA, Whitehall VL, Spring KJ, Wynter CV, Walsh MD, Barker MA, Arnold S, McGivern A, Matsubara N, Tanaka N, Higuchi T, Young J, Jass JR and Leggett BA: BRAF mutation is associated with DNA methylation in serrated polyps and cancers of the colorectum. Gut 53: 1137-1144, 2004

29. Yang S, Farraye FA, Mack C, Posnik O and O'Brien MJ: BRAF and KRAS mutations in hyperplastic polyps and serrated adenomas of the colorectum: relationship to histology and $\mathrm{CpG}$ island methylation status. Am J Surg Pathol 28: 1452-1459, 2004.

30. Yantiss RK, Panczykowski A, Misdraji J, Odze RD, Rennert H and Chen Y-T: A comprehensive study of non-dysplastic and dysplastic serrated polyps of the vermiform appendix. Am J Surg Pathol 31: 1742-1753, 2007.

31. Fogt F, Vortmeyer AO, Goldman H, Giordano TJ, Merino MJ and Zhuang Z: Comparison of genetic alterations in colonic adenoma and ulcerative colitis-associated dysplasia and carcinoma. Hum Pathol 29: 131-136, 1998.

32. Gradowski JF, Mantha GS, Hunt JL and Dacic S: Molecular alterations in atypical adenomatous hyperplasia occurring in benign and cancer-bearing lungs. Diagn Mol Pathol 16: 87-90, 2007. 